\title{
Dermoscopic features of pseudoxanthoma elasticum
}

\section{Aida Oulehri, Zakia Douhi, Sara Elloudi, Hanane Baybay, Fatima Zahra Mernissi}

\author{
Department of Dermatology, University Hospital Hassan II, Fez, Morocco
}

Corresponding author: Aida Oulehri, MD, Email: aidaoulehri@gmail.com

\begin{abstract}
Pseudoxanthoma elasticum (PXE), also known as Gröenblad-Strandberg syndrome, is an autosomal recessive connective tissue disease characterized by aberrant mineralization of soft connective tissue resulting in fragmentation of elastic fibers, involving primarily the skin, eyes and cardiovascular. To our knowledge, there is only two other reports of the dermoscopic features of PXE in the literature. We report clinical and dermoscopic description of two new cases. We hypothesize for the first time that the dermoscopic appearance may differ according to the age of PXE lesions.
\end{abstract}

Key words: Dermoscopy; Pseudoxanthoma elasticum; Yellowish areas; Reticulated vessels

\section{INTRODUCTION}

Pseudoxanthoma elasticum (PXE) is an inherited disease characterized by aberrant mineralization of soft tissue and fragmentation of elastic fibres [1]. However, the pathophysiology, particularly the mechanism of ectopic mineralization remains largely unknown. PXE, as other genodermatoses, is currently an intractable disease, associated with considerable morbidity and occasional mortality due to cardiovascular complications [2]. Cutaneous lesions consist of small, asymptomatic, yellowish papules or larger coalescent plaques, typically located on the neck and the flexural areas [3]. The histological examination of cutaneous lesions, revealing accumulation of pleomorphic elastic structures in mid dermis, is essential for the definitive diagnosis of PXE. Cutaneous findings present mainly a cosmetic issue, but they also allow the identification of the disease and thus the possibility of monitoring the risks of ocular and cardiovascular complications, which are associated with considerable morbidity and mortality [4].

\section{CASE REPORT}

Patient 1 was a 30-year-old woman, who consulted for persistent asymptomatic skin lesions on his neck, who started at the age of 14 years old, extending progressively to the rest of the body. Physical examination revealed confluent yellowish papules with cobblestone appearance over the entire neck, bilateral axillae, under mammary and inguinal folds (Figs. la and lb). The affected skin was typically lax, wrinkled, and redundant (Figs. lc and ld). Dermoscopic findings included bright purple background, multiple irregular yellowish areas alternating with subtle reticulated vessels. The yellowish areas coalesced to form parallel strands or a pseudo network (Figs. 2 - 4). Histopathological examination of a skin biopsy stained with haematoxylin and eosin (H\&E) showed preserved morphology of the epidermal, the superficial, medium and deep dermis is encumbered with elastic clumped and irregularly packed fibers. They are basophilic and sometimes mineralized. These fibers colored in black by Orcein are intimately mixed with collagen fibers without any abnormality. Based on these findings, we diagnosed the patient with PXE.

Patient 2 was a 21 -year-old young woman, who presented with asymptomatic skin lesions of her neck. These lesions had appeared at 15 and had since, slowly enlarged. Her medical history included scalp psoriasis. On physical examination, we observed multiple yellowish and confluent papules symmetrically distributed over the patient's entire neck (Figs. 5a and $5 \mathrm{~b}$ ). Without other locations on the rest of the body. Dermoscopic findings included coalescing and reticulated yellow-white areas on a light purple-

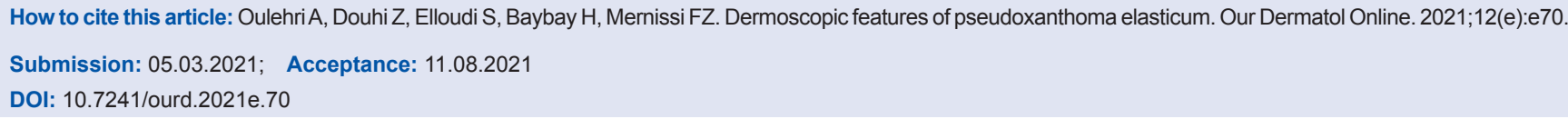




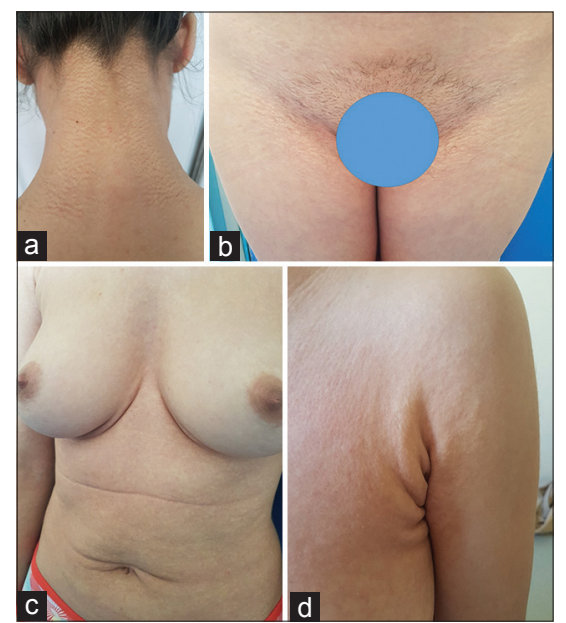

Figure 1: Patient 1. Confluent yellowish papules with cobblestone appearance on the entire neck (a) and inguinal folds (b), the affected skin was typically lax, wrinkled, and redundant under mammary (c) and on bilateral axillae (d).

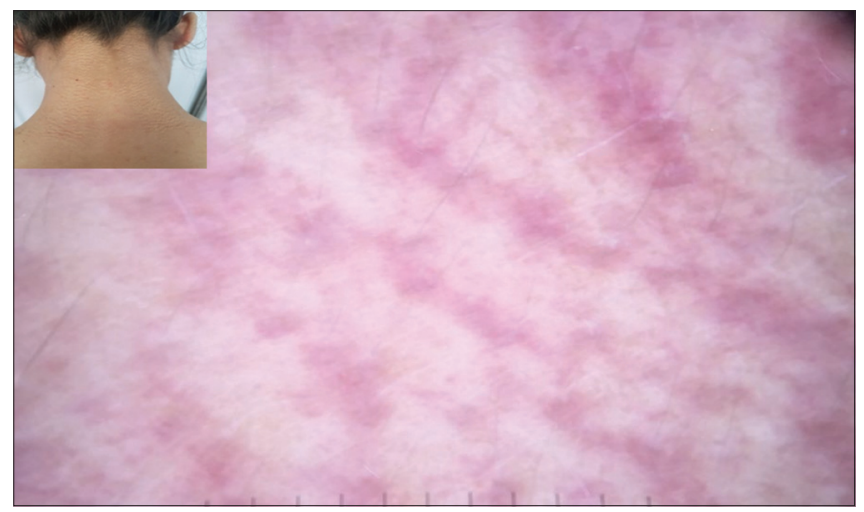

Figure 2: Dermoscopic findings included bright purple background, multiple irregular yellowish areas coalescing to form parallel strands.

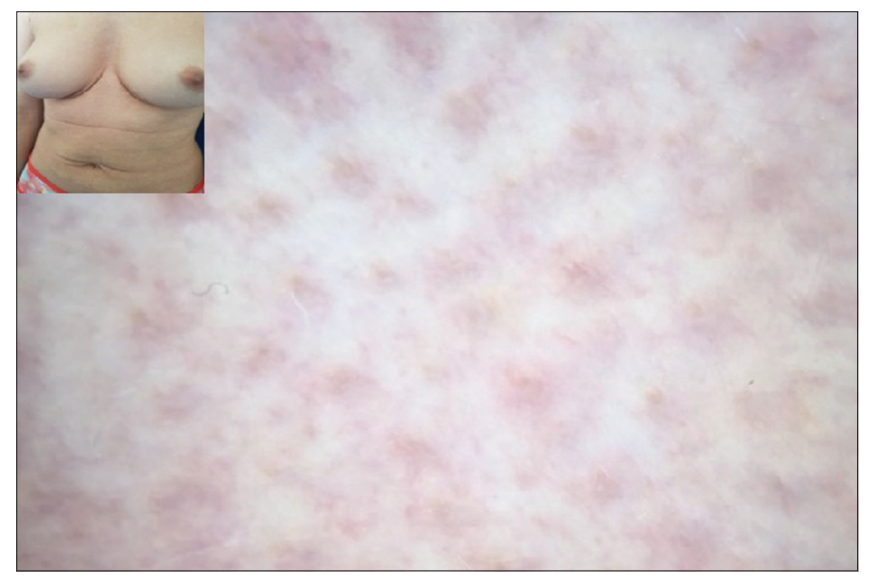

Figure 3: Dermoscopic findings included purplish background, multiple irregular yellowish areas coalescing to form a pseudo network.

red background along with prominent reticulated and linear vessels (Figs. 6 and 7). Histopathological examination of a skin biopsy stained with haematoxylin and eosin (H\&E) showed the same aspect described in

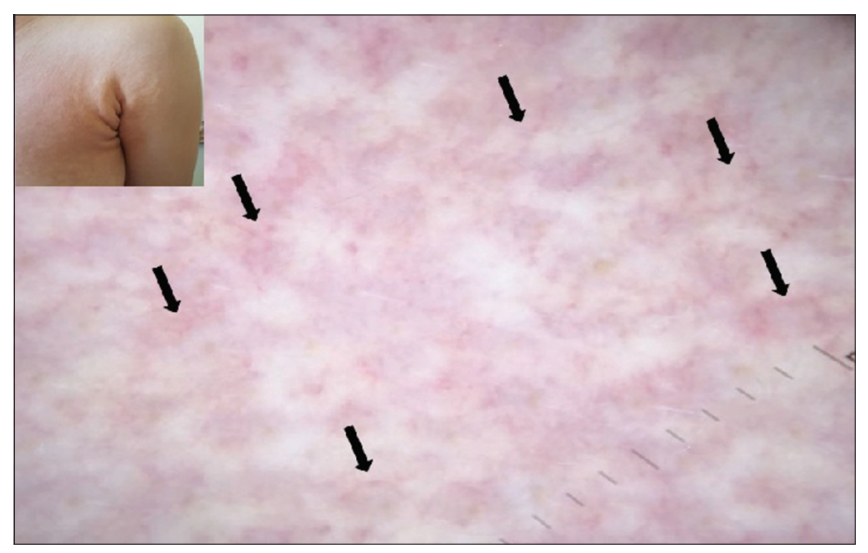

Figure 4: Dermoscopic findings included purplish background, multiple irregular yellowish areas alternating with subtle reticulated vessels (black arrows).

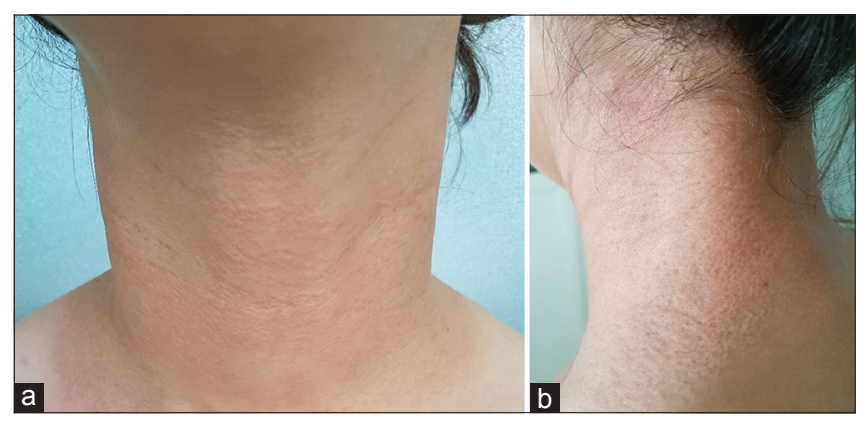

Figure 5: $(a, b)$ Patient 2. Multiple yellowish and confluent papules symmetrically distributed over the patient's entire neck.

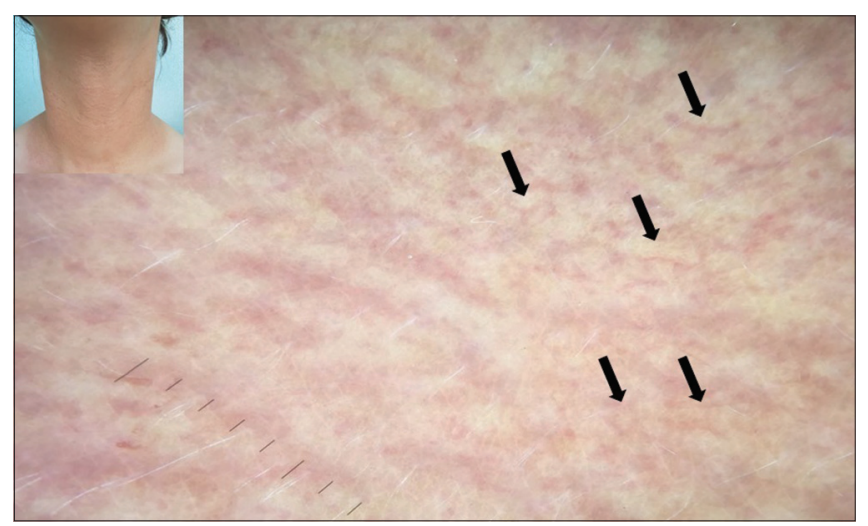

Figure 6: Dermoscopic findings included coalescing and reticulated yellow-white areas on a light purple-red background along with prominent reticulated and linear vessels (black arrows).

the first patient, we therefore diagnosed the patient with PXE.

\section{DISCUSSION}

To our knowledge, there is only two other reports of the dermoscopic features of PXE in the literature. Lacarrubba et al. [5] reported them as multiple 


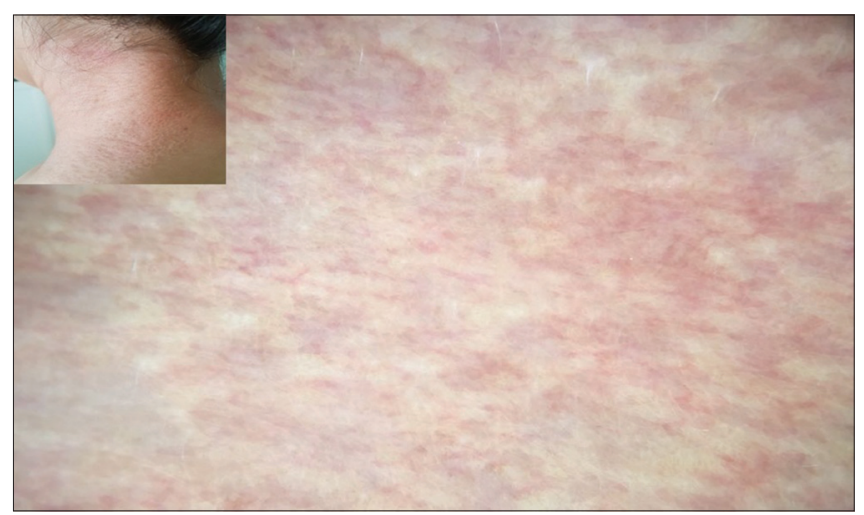

Figure 7: Dermoscopic findings included coalescing and reticulated yellow-white areas on a light purple-red background along with prominent reticulated and linear vessels (black arrows).

irregular, yellowish areas coalescing to parallel strands alternating with prominent linear vessels. Kawashima et al. [4] reported the dermoscopic features of PXE as coalescing and reticulated yellow-white clods on a light purplish-red background along with reticulated vessels. The dermoscopic description of our two patients is very close to that of the literature.

Lacarrubba et al. [5] have suggested that the prominent superficial linear vessels may be caused by a vascular rearrangement driven by the underlying dermal elastolysis. Kawashima et al. [4] have suggested that the purplish-red background is due to mid-dermal vasodilation. Both authors emphasized that the yellowish-white area in PXE may reflect the pathological features of degeneration and/or fragmentation of elastic fibres andcalcium deposits in the mid dermis.

There were several differences between our two cases; the background was purplish or bright purple for patient one and purple red for patient two. Patient one showed the predominance of confluent yellowish areas; on the other hand, patient two presented the predominance of vascular structures, which were much more discreet in patient one.

We hypothesized that these results could be explained by the stage of evolution of PXE. In fact, the first patient who presented old skin lesions evolving for 16 years presented on the dermoscopic level the predominance of the fibrillar component, while the second patient, who had a much shorter duration of course of the disease and skin lesions which had only progressed for six years, showed the predominance of vascular lesions on dermoscopy.

\section{CONCLUSION}

We add two new dermoscopic description cases of PXE, We hypothesize for the first time that the dermoscopic appearance may differ according to the age of PXE lesions, of course other case reports or series are necessary in order to confirm these data and to be able to use the dermoscope as a non-invasive tools for diagnosis and monitoring of PXE.

\section{Consent}

The examination of the patient was conducted according to the principles of the Declaration of Helsinki.

The authors certify that they have obtained all appropriate patient consent forms, in which the patients gave their consent for images and other clinical information to be included in the journal. The patients understand that their names and initials will not be published and due effort will be made to conceal their identity, but that anonymity cannot be guaranteed.

\section{REFERENCES}

1. Marconi B, Bobyr I, Campanati A, Molinelli E, Consales V, Brisigotti V, et al. Pseudoxanthoma elasticum and skin: Clinical manifestations, histopathology, pathomechanism, perspectives of treatment. Intractable Rare Dis Res. 2015;4:113-22.

2. Roach ES, Islam MP. Pseudoxanthoma elasticum. Handb Clin Neurol. 2015;132:215-21.

3. Chassaing N, Martin L, Calvas P, Le Bert M, Hovnanian A. Pseudoxanthoma elasticum: a clinical, pathophysiological and genetic update including 11 novel ABCC6 mutations. J Med Genet. 2005;42:881-92.

4. Kawashima S, Togawa Y, Miyachi H, Matsue H. Dermoscopic features of pseudoxanthoma elasticum. Clin Exp Dermatol. 2018;43:175-9.

5. Lacarrubba F, Verzì AE, Caltabiano R, Micali G. Dermoscopy of pseudoxanthoma elasticum. J Am Acad Dermatol. 2017;76:S69-S70.

Copyright by Aida Oulehri, et al. This is an open-access article distributed under the terms of the Creative Commons Attribution License, which permits unrestricted use, distribution, and reproduction in any medium, provided the original author and source are credited.

Source of Support: Nil, Conflict of Interest: None declared. 